\title{
The nursing techniques and technologies
}

Maria Aparecida de Luca Nascimento ${ }^{1}$

Professions are characterized by their technical elements. Thus, they are socially known and perpetuated by their practices. With the nursing profession, it could not be different, and it is characterized by care actions.

By considering that the nursing care process is multifaceted and multidimensional, and that several sciences are focused on it, this care, due to the fact that it is provided to the human being, who is a social being, is underpinned by the biological, human and social sciences.

Nursing was raised to scientific levels in England, during the mid-XIX century, from the precepts of Florence Nightingale, more specifically, through the nursing procedures during the care process, including in this care, the macro and microenvironment where this care was performed. However, it was not until the beginning of the XX century that Nursing emerged in the United States as a systematized and organized way of providing care to the patient, also structuring its knowledge through the development of skills in performing technical procedures.

Thereafter, Nursing was solidified as a profession, thereby enabling its professionals to exercise it through care, in all its complexity; of teaching, in its entire dimension, and of extension, in its entire coverage.

Nevertheless, one can observe that, despite all the importance credited to the care process, in its widest meaning, the scientific papers that deal with it are scarce, when compared to other themes related to the several procedures developed by the nursing professionals.

The technical procedures require of those who practice it elements such as speed, training, common sense, and theoretical-practical knowledge. However, this practicality cannot be confused with rationality deprived of sensitivity, the same that triggers the act of caring for, often, from a non-verbal communication.

In the XX century, Emerson Merhy classified the technologies into light, hard-light and hard, and considered them as a set of ideas, structuring of protocols and artifacts, respectively.

After that, the scientific productions of nursing that deal, for example, with the creation of several artifacts designed for the daily practice of care, the enunciation of algorithms for the insertion of venous catheters, the elaboration of strategies for positioning and the eliminating pain newborn, the design of a dressing capable of healing pharyngocutaneous fistula in laryngectomized patients, and several other ways of care, prove that we deal with the most different types of technologies. In this manner, by considering that all these productions have emerged from the act of caring for, when they are intrinsically linked to the act of doing, demonstrate all the scientificity contained in this daily practice of the nursing staff.

Many papers produced and patented by the hospital materials and equipment industries derive from the product of discharge of artifacts improvised by the nursing staff during its care actions, thereby not earning the profits that could be generated from their true idealizers.

The improvisations and adaptations of materials and artifacts that emerge during the act of caring for emerge from the lack of suitable material for the work and are the source for intellectual production. Accordingly, one can insert the displeasure of the professional in this aspect, and, consequently, its disposal.

\footnotetext{
${ }^{1}$ Universidade Federal do Estado do Rio de Janeiro. Rio de Janeiro, RJ, Brazil. 
Nonetheless, there is a need to lift up the flag of the entrepreneurship teaching in the nursing courses, so that the process of caring for performed by the nursing professional is viewed as a large field of experimentation of prototypes, and so that the nursing profession is admittedly constructed on the basis of the science-technology binomial. 